\title{
Optimizing Mask Ventilation: Literature Review and Development of a Conceptual Framework
}

\author{
Daniel Saddawi-Konefka MD MBA, Susan L Hung MD, Robert M Kacmarek PhD RRT FAARC, \\ and Yandong Jiang MD PhD
}

\author{
Introduction \\ Definition and Epidemiology \\ Conceptual Framework for Difficult \\ Mask Ventilation \\ Inadequate Seal at the Mask-Patient Interface \\ Increased Airway Resistance \\ Decreased Distal Compliance \\ Summary Recommendations for the Emergency Situation \\ Summary
}

\begin{abstract}
Mask ventilation is lifesaving, especially in cases of difficult intubation. Many publications have offered distinct techniques for optimizing mask ventilation. This article reviews currently available difficult mask ventilation literature and theory. We divide difficult mask ventilation into 3 broad categories based on etiology: inadequate mask seal, increased airway resistance, and decreased respiratory compliance. Published strategies for overcoming difficulty are presented and organized by etiology. Key words: positive-pressure respiration; pulmonary ventilation; respiratory therapy; respiration; critical care; anesthesiology. [Respir Care 2015;60(12):1834-1840. (C 2015 Daedalus Enterprises]
\end{abstract}

\section{Introduction}

Maintaining adequate oxygenation in an apneic patient is paramount to patient safety. Failure to oxygenate, not

\footnotetext{
Dr Saddawi-Konefka is affiliated with the Department of Anesthesia, Critical Care and Pain Medicine, and Dr Kacmarek is affiliated with the Department of Respiratory Care, Massachusetts General Hospital, Harvard Medical School, Boston, Massachusetts. Dr Hung is affiliated with Westchester Anesthesiologists, White Plains Hospital, White Plains, New York. Dr Jiang is affiliated with the Department of Anesthesiology, Vanderbilt University, Nashville, Tennessee.

Dr Kacmarek has disclosed relationships with Covidien and Venner Medical. The other authors have disclosed no conflicts of interest.

Correspondence: Yandong Jiang MD PhD, Department of Anesthesiology, Vanderbilt University Medical Center, 1301 Medical Center Drive, 4648 TVC, Nashville, TN 37232-5614. E-mail: yandong.jiang@ vanderbilt.edu.
}

DOI: $10.4187 /$ respcare.04183 failure to intubate, ultimately leads to brain injury and cardiovascular collapse. ${ }^{1}$ Mask ventilation is the most fundamental technique in maintaining oxygenation and can avoid catastrophic events, even when intubation is unsuccessful. Developing skills to optimize mask ventilation is therefore crucial for caregivers who find or render patients apneic, particularly anesthesia providers, emergency medicine clinicians, emergency medical service personnel, and respiratory therapists.

Despite the importance of mask ventilation and the correlation between difficult mask ventilation and difficult intubation, ${ }^{1}$ the mechanism of failed mask ventilation is not as extensively investigated as that of failed intubation. The majority of the literature on techniques to improve mask ventilation is dated, and fewer devices are available to facilitate mask ventilation than are available to facilitate intubation. Although difficult intubation may not be as challenging as in the past due to the availability of video laryngoscopes, the incidence of major complications related to airway management has not decreased. ${ }^{2}$ 
This review presents a conceptual framework for the etiologies of difficult mask ventilation based on available literature. It subsequently details appropriate etiology-specific management strategies. We focus on skills and resources readily available to clinicians. Although it is unlikely that all difficult mask ventilation can be eliminated, we hope that this working framework will provide clinicians a more simplified approach to difficult mask ventilation situations, expand the clinician's repertoire of techniques to optimize mask ventilation, and help reduce the impact of difficult mask ventilation and its related complications.

\section{Definition and Epidemiology}

A standard, widely accepted, objective definition for difficult mask ventilation does not exist, and application of available definitions is complicated by interoperator variability. These issues complicate communication of patient history, reporting of epidemiologic data, and performance of objective studies on difficult mask ventilation. Most published definitions are binary (ie, difficult or not difficult) and include various aspects of inadequate oxygenation and/or ventilation. Just as with intubation, patients more often fall on a spectrum between easy and impossible mask ventilation. Therefore, a graded definition as we commonly use for intubation is likely more useful clinically. Although none have gained widespread acceptance, such classification systems have been proposed. A 2004 classification and description scale developed by Han et $\mathrm{al}^{3}$ suggests grades $0-4$ : grade $0=$ mask ventilation not attempted, grade $1=$ ventilation by mask, grade $2=$ ventilation by mask with adjuvant, grade $3=$ difficult mask ventilation (inadequate, unstable, or requiring 2 practitioners), and grade $4=$ unable to ventilate.

Reported epidemiologic data suggest that difficult mask ventilation occurs in $1.4 \%$ (range $0.9-7.8 \%$ ) of patients undergoing general anesthesia and $4-11 \%$ of patients in the emergency room. ${ }^{4,5}$ The actual rate of difficult mask ventilation in field resuscitation is unknown and likely higher than that encountered in the hospital given the suboptimal conditions. Predictive factors include high body mass index, edentulous and/or bearded patients, and high Mallampati class. Impossible mask ventilation is less predictable, with estimates of $0.07-0.16 \% .^{2}$ With 21 million general anesthesia procedures performed annually in the United States, this equates to $\sim 15,000-34,000$ impossible mask ventilations per year. As the obese and morbidly obese populations increase, it is reasonable to speculate that these numbers will grow, and difficult mask ventilation will remain challenging to health-care providers.

\section{Conceptual Framework for Difficult Mask Ventilation}

Difficult mask ventilation may be caused by patientrelated or non-patient-related factors. Although they are important, non-patient-related factors, such as operator inexperience or faulty equipment, will not be the focus of this review.

Successful mask ventilation involves the flow of gas from a positive-pressure source to the patient's lungs via a mask-patient interface, such as a face mask. The flow of gas along a path is inversely related to resistance and directly related to the decrease in pressure along that path. It follows that difficult mask ventilation must be caused by one or more of: (1) a low-resistance alternative path due to an inadequate seal, (2) an increase in air-flow resistance along the path to the lungs, or (3) a decrease in compliance of the lungs and/or chest wall leading to increased distal pressure. Understanding the etiology of difficult mask ventilation is essential to making the appropriate diagnosis and subsequent management in the emergency situation. Below, we outline an approach to difficult mask ventilation based on this model. A summary of underlying causes and recommended practices discussed below is presented in Tables 1 and 2.

\section{Inadequate Seal at the Mask-Patient Interface}

A gas will flow discriminately to lower-resistance paths. An inadequate seal at the mask-patient interface provides a lower-resistance escape for positive-pressure breaths. Similarly, an increased airway resistance will make alternative paths (eg, the mask-patient interface or esophagus) more likely to receive flow. Factors that contribute to a poor seal at the mask-patient interface include improper mask size, shape/design or positioning, facial hair, edentulism, micrognathia, maxillomandibular deformities, and foreign objects (eg, nasogastric tubes). ${ }^{6}$

Independent of the cause of inadequate seal, higher fresh gas flows may help compensate for the leak (when using a valveless systems [Mapleson] or anesthesia ventilators). In the more commonly found bag-valve-mask systems, increasing flow does not compensate for a leak unless a PEEP valve is applied. Even when possible, compensation is limited because most operating room ventilators maximally deliver $12 \mathrm{~L} / \mathrm{min}$. This can be improved by using the oxygen flush feature available on all ventilators. ${ }^{6}$ Although not generally available in the operating room, the use of a ventilator designed for noninvasive ventilation or the use of ICU ventilators with leak compensation can dramatically improve ventilation. Many of these ventilators are designed to compensate for leaks up to $60 \mathrm{~L} / \mathrm{min}^{7,8}$

Face masks are available in different shapes, and some can be reshaped to optimize fit to the patient's face. The 
Table 1. Causes of Difficult Mask Ventilation Grouped by Conceptual Etiology

\begin{tabular}{|c|c|}
\hline Etiology & Contributing Factors \\
\hline \multirow[t]{6}{*}{ Inadequate mask seal } & Improper mask size \\
\hline & Inappropriate mask shape/design \\
\hline & Facial hair \\
\hline & Edentulism \\
\hline & $\begin{array}{l}\text { Maxillomandibular deformities (eg, } \\
\text { micrognathia) }\end{array}$ \\
\hline & Foreign objects (eg, nasogastric tube) \\
\hline \multirow{16}{*}{$\begin{array}{l}\text { Increased airway } \\
\text { resistance }\end{array}$} & Upper airway \\
\hline & Tonsillar and adenoidal hypertrophy \\
\hline & Redundant soft tissue \\
\hline & Large tongue or epiglottis \\
\hline & Airway edema \\
\hline & Oropharyngeal tumors \\
\hline & $\begin{array}{l}\text { External compression (eg, large neck mass } \\
\text { or hematoma) }\end{array}$ \\
\hline & Laryngospasm \\
\hline & Lower airway \\
\hline & Airway secretions \\
\hline & Excessively applied cricoid pressure \\
\hline & Foreign body \\
\hline & Tracheomalacia \\
\hline & Tracheal stenosis \\
\hline & Airway or mediastinal mass \\
\hline & Bronchospasm \\
\hline \multirow{7}{*}{$\begin{array}{l}\text { Decreased respiratory } \\
\text { compliance (either } \\
\text { of the lungs and/or } \\
\text { chest wall) }\end{array}$} & $\begin{array}{l}\text { Restrictive lung disease (eg, pulmonary } \\
\text { fibrosis) }\end{array}$ \\
\hline & $\begin{array}{l}\text { Chest wall deformity (eg, severe } \\
\text { kyphoscoliosis) }\end{array}$ \\
\hline & Obesity \\
\hline & Abdominal compartment syndrome \\
\hline & Pregnancy \\
\hline & External compression (eg, orthotics) \\
\hline & Tension pneumothorax \\
\hline
\end{tabular}

routinely recommended face mask placement fits the bridge of the nose superiorly, the nasolabial folds laterally, and the mental crease inferiorly. The smallest appropriate mask is recommended to reduce dead space and eye compression. The top of a well-seated mask should be slightly inferior to the bridge of the nose to avoid leak into the eyes. Several methods of holding a face mask to achieve adequate seal have been described, including the commonly employed C-E clamp, where the thumb and index finger form a $\mathrm{C}$ shape over the mask while the third, fourth, and fifth fingers (the E) pull the mandible into the mask. With the double C-E technique, each thumb and index finger form a $\mathrm{C}$ shape over each side of the mask while the third, fourth, and fifth fingers of both hands lift the mandible toward the mask. Another 2-handed technique, the V-E technique, uses a thumb and thenar eminence over each side of the mask while the second through fifth digits pull the jaw upward (Fig. 1). In another one- handed technique, known as the $\mathrm{V}$ (or $\mathrm{N}$ ) technique, the hand is rotated such that the care provider's wrist is at the mental protuberance. The thumb is placed on one side of the mask, the second through fourth fingers are placed on the opposite side of the mask, and the fifth finger lifts the mandible at the mental protuberance.

Compared with one-handed ventilation, 2-handed approaches provide better seal ${ }^{9,10}$ and produce greater tidal volumes $^{11,12}$ but require an additional provider or a machine to deliver the positive-pressure breaths. ${ }^{13}$ Although possibly underutilized, setting a mechanical ventilator to deliver positive-pressure breaths while masking can be advantageous. It frees both hands for mask application and provides additional diagnostic information (eg, the pressure tracing from a single breath can differentiate a circuit leak from a resistance or compliance problem). Setting peak pressures to $<20 \mathrm{~cm} \mathrm{H}_{2} \mathrm{O}$ decreases the risk of inadvertent gastric insufflation. Although newer evidence suggests that $15 \mathrm{~cm} \mathrm{H}_{2} \mathrm{O}$ may cause less gastric insufflation, it might not be high enough to produce effective mask ventilation when difficult mask ventilation is encountered. ${ }^{14,15}$

Facial hair and edentulism are 2 commonly encountered causes of inadequate seal. Facial hair can be shaved, although it is often undesired by patients and may not be feasible in emergency situations. Alternatively, large occlusive dressings can be placed over facial hair to provide an adequate surface for a seal. This has been described with clear adhesive tape (such as Tegaderm, 3M, St Paul, Minnesota), plastic wrap, gel, gauze, and even defibrillator pads. ${ }^{16,17}$ Placing the inferior aspect of the mask inside the lower lip at the alveolar ridge has also been described. ${ }^{16}$ Edentulism leads to atrophy of bone and buccinator muscles. This loss of structure creates a gap between the cheeks and the mask. ${ }^{18,19}$ Using 2 hands to pull the patient's cheeks to oppose the mask during mask ventilation often helps minimize leak. Recreating structure can be achieved by leaving the dentures in place ${ }^{18}$ or by packing the patient's cheeks with gauze, ${ }^{20}$ although the latter risks foreign object obstruction or aspiration.

In cases in which an inadequate face-mask seal persists despite optimization strategies, alternative mask-patient interfaces can help. A nasal mask or a toddler-sized mask with the lower border resting above the patient's upper lip can provide adequate seal if the patient's mouth is occluded with a hand or dressing..$^{21}$ The nasal mask may also relieve airway obstruction as described in the next section. The double nasopharyngeal tube, which delivers positive pressure directly to the pharyngeal cavity, ${ }^{22}$ is no longer commercially available, but successful utilization of an ordinary nasopharyngeal airway connected to an endotracheal tube adapter while compressing the contralateral nostril has been described. ${ }^{23}$ Supraglottic airways provide another patient interface that is discussed further below. 
Table 2. Summary of Recommended Practices for Difficult Mask Ventilation

\begin{tabular}{llc}
\hline \hline \multicolumn{1}{c}{ Inadequate Mask Seal } & Increased Airway Resistance & Decreased Respiratory Compliance \\
\hline General principles & General principles & General principles \\
Increase gas flows to overcome leak & Positioning & Ensure appropriate anesthetic depth or paralysis \\
Try different mask size or shape & Sniffing position & Increase driving pressures \\
Try 2-handed mask & Jaw thrust & Reverse Trendelenburg \\
Consider nasal mask & Chin lift & Tension pneumothorax \\
Move seal into nose or mouth (see devices & Reverse Trendelenburg & Emergent needle decompression \\
under "Increased Airway Resistance") & Pharmacologic & Consider deepening anesthetic or paralyzing \\
Consider an alternative ventilator designed & Paracentesis for fluid \\
for noninvasive ventilation with leak & Devices & Laparotomy to release hypertension in emergency \\
compensation & Oral/nasal airway & Pregnancy \\
Facial hair & Laryngeal mask airway & Operative delivery of the fetus in emergency \\
Shave or cover beard with occlusive & Pharyngeal endotracheal tube & \\
material (eg, gel, Tegaderm) with hole & Increase driving pressures or apply CPAP & \\
over mouth & Consider nasal mask & \\
Edentulism & Lower-airway etiologies & \\
Leave dentures in, pull cheeks anteriorly, & Consider suctioning & \\
or pack cheeks & Remove foreign bodies or displace into distal & \\
Foreign bodies & branch & \\
Consider removing (eg, remove nasogastric & Treat bronchospasm & \\
tube) & & \\
\hline Determine likely etiologies or consider addressing nonspecifically with general principles. & \\
\hline & & \\
\hline
\end{tabular}

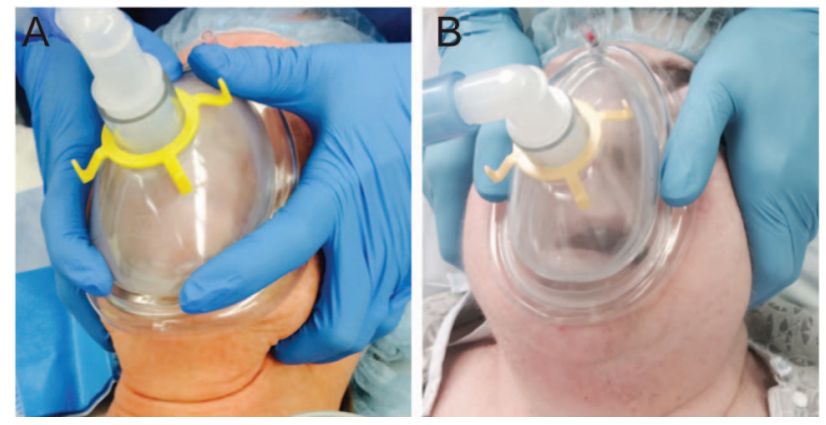

Fig. 1. Two-hand bag-mask ventilation techniques. A: With the double C-E technique, each thumb and index finger form a $\mathrm{C}$ shape over each side of the mask while the third, fourth, and fifth fingers of both hands lift the mandible toward the mask. B: With the V-E technique, the thumbs and thenar eminence are placed over each side of the mask while the second through fifth digits pull the jaw upward (Clear Comfort air cushion face mask, Hudson $\mathrm{RCl} /$ Teleflex Medical, Research Triangle Park, North Carolina).

\section{Increased Airway Resistance}

Increased resistance is most commonly due to supraglottic issues, although infraglottic contributions to airflow resistance (which are typically more difficult to circumvent) are discussed at the end of this section. ${ }^{6}$ The upper airway is composed primarily of soft tissue, bordered proximally by the bones and cartilage of the nose and distally by the rigid trachea. The transitional area of unsupported soft tissue or pharyngeal tube is prone to collapse, especially when the upper-airway dilator mus- cles, such as the genioglossus, are depressed due to loss of consciousness or pharmacologic paralysis. This is further compromised by tonsillar and adenoidal hypertrophy, redundant soft tissue (eg, morbid obesity or obstructive sleep apnea), large tongue or epiglottis, airway edema (eg, after repeated intubation attempts, trauma, or angioedema), oropharyngeal tumors, external compression (eg, large neck masses or hematoma), or laryngospasm. To mask-ventilate when an upper-airway obstruction is present, the generated positive pressure must overcome the critical closing pressure of the collapsed pharynx, ${ }^{24}$ or the obstructed airway can be bypassed with placement of an airway adjuvant or supraglottic airway device.

Positioning maneuvers can reduce upper-airway obstruction. The sniffing position (lower-cervical flexion and upper-cervical extension) and chin lift stent open pharyngeal soft tissue by increasing longitudinal tension. Jaw thrust displaces the mandible, ${ }^{25-27}$ increasing the retrolingual and retropalatal spaces by pulling the tongue anteriorly, although this benefit may be less present in the obese patient. ${ }^{26}$ With a reverse Trendelenburg position, gravity pulls down the diaphragm and trachea, stretching the pharyngeal tube and decreasing its collapsibility. ${ }^{28}$ Application of CPAP increases airway patency by maintaining the outward pressure gradient in the pharyngeal cavity. ${ }^{29} \mathrm{CPAP}$ also has an added benefit of increasing lung volume, which improves oxygenation and provides longitudinal tension on the pharyngeal tube..$^{30}$ Nasal mask ventilation provides pressure that anteriorly displaces the tongue and palate, and it may be more effective than combined oronasal ven- 

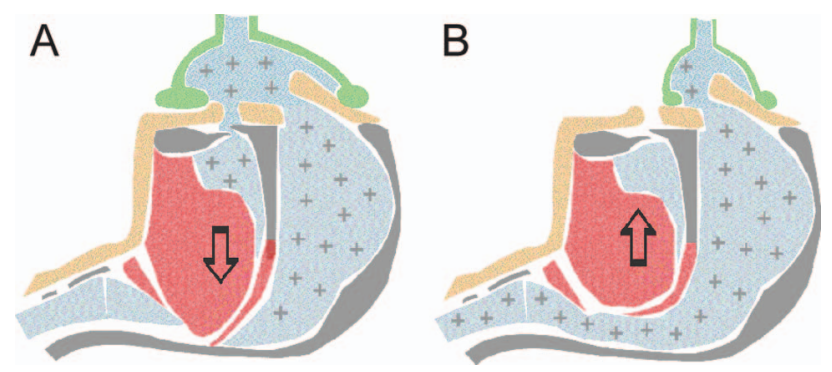

Fig. 2. Nasal mask ventilation provides pressure that anteriorly displaces the tongue and palate $(\mathrm{B})$, and it may be more effective than combined oronasal ventilation, which can posteriorly displace the tongue and palate and worsen obstruction (A).

tilation, which can posteriorly displace the tongue and palate and worsen obstruction (Fig. 2). It provides larger tidal volumes with lower peak inspiratory airway pressures $^{31,32}$ but has not been rigorously evaluated in patients with known difficult mask ventilation.

When airway obstruction is caused by active pharyngeal tube closure (eg, light anesthesia, laryngospasm, opioidinduced vocal cord closure), ${ }^{33}$ deepening anesthesia or pharmacologic paralysis helps. ${ }^{34-36}$ Of course, before paralyzing a patient, proper airway equipment and a practitioner experienced in advanced airway management should be present. Historically, muscle relaxants were not administered until confirmation of adequate mask ventilation because of the theoretical ability to restore spontaneous breathing. ${ }^{37}$ Many now believe that patients may not regain spontaneous ventilation before life-threatening hypoxemia arises. The other, possibly more important part of this controversy is that neuromuscular blockade will often help overcome the difficulty in mask ventilation, and delaying its administration may introduce unnecessary risk. Currently, the administration of muscle relaxant in cases of suspected difficult or impossible mask ventilation remains controversial.

Bypassing an obstruction with adjuvant devices, such as an oral or nasal airway, or supraglottic devices should always be an early consideration. A correctly sized oral or nasal airway often extends from the corner of the mouth or nose to just above the angle of the mandible. In practice, finding a perfect fit is often challenging, even in experienced hands. ${ }^{38}$ An adjuvant airway that is too small can posteriorly displace the tongue, worsening obstruction, and one that is too long may inadvertently intubate the esophagus or posteriorly displace the epiglottis. ${ }^{38}$ Dental damage is a risk with oral airway insertion (especially in the presence of poor dentition), and epistaxis, which may further complicate a difficult airway, is a risk with nasal airways. ${ }^{6}$ Gentle insertion and adequate nasal airway lubrication may mitigate these complications. Laryngeal mask airways are the most popular supraglottic devices.
Optimal positioning, which excludes the esophagus and epiglottis, is achieved only $45-60 \%$ of the time. ${ }^{39}$ Suboptimal placement may result in partial obstruction, but gas flow is often sufficient. Complete failure to adequately ventilate through a laryngeal mask airway is reported in $0.4-6 \%$ of cases and may result from complete obstruction by the epiglottis, a rotated laryngeal mask airway, or an inadvertently bent laryngeal mask airway tip. Laryngeal mask airways are more difficult to place in patients with small mouths, large tongues, large tonsils, or posterior larynges. In such cases, a laryngoscope may facilitate placement. When a laryngeal mask airway is not available or unsuccessful, placing an endotracheal tube in the supraglottic area and then hyperinflating the cuff with 50-60 $\mathrm{mL}$ (the poor man's laryngeal mask airway) has been described as a successful rescue technique. ${ }^{40-42}$

Infraglottic causes of increased air-flow resistance are less commonly encountered but are important to consider as the management is etiology-specific. Causes include airway secretions or mucous plugs, foreign bodies, excessively applied cricoid pressure, bronchospasm, tracheomalacia, tracheal stenosis, and airway or mediastinal mass. Airway secretions can be suctioned. Foreign bodies can be removed with flexible or rigid bronchoscopy or, in the setting of complete airway obstruction, may need to be pushed distally into a branch. Bronchospasm is treated with $\beta$ agonists such as albuterol, increased concentrations of volatile anesthetics, or increased PEEP. Intravenous epinephrine should also be considered because inhaled medications may not reach the intended target with severe bronchospasm. Tracheomalacia, tracheal stenosis, and airway or mediastinal tumors are traditionally managed by maintaining spontaneous ventilation and appropriate positioning, although CPAP may also help by increasing luminal pressure and lung volume. In the case of a fixed obstruction, increasing the driving pressure and lengthening inspiratory time assist in ventilating past the obstructions. These patients are often able to breathe with only minimal difficulty before induction of anesthesia, but as lung volume is reduced, partial occlusion of the lower airway becomes complete obstruction. Preoperative examination should identify the position in which the patient is able to breathe most easily. The patient should be placed in this rescue position if impossible ventilation develops after induction. In some cases, a rigid bronchoscope may be required to bypass the obstructed segment. In severe cases, emergency sternotomy and mass elevation may be necessary to avoid death due to impossible ventilation. ${ }^{43}$ Referral to a tertiary care center with extracorporeal membrane oxygenation capabilities should be considered if the likelihood of impossible mask ventilation and intubation is high. 


\section{Decreased Distal Compliance}

Decreased compliance of the lungs and chest wall causes difficult mask ventilation by increasing distal pressure, which decreases the driving-pressure gradient. Contributing factors include inadequate depth of anesthesia or inadequate paralysis (ie, ventilation asynchrony), large body habitus, restrictive lung disease (which may be chronic, as with kyphoscoliosis, or acute, as with ARDS), intra-abdominal hypertension, external compression (eg, a compressing orthotic), and tension pneumothorax. ${ }^{6}$ Treatment includes ensuring appropriate anesthetic depth or paralysis and provision of pressures sufficient to ventilate appropriately or accepting the presence of hypercapnia, as is often done in the setting of the ARDS. The pressures necessary will be high, which may exacerbate leak at the maskpatient interface and may increase risk of gastric insufflation. However, the transpulmonary pressures will remain normal given the decreased compliance unless lung compliance alone is decreased (eg, ARDS). Externally compressing devices should be removed when possible, and tension pneumothorax should be promptly treated with needle decompression because positive pressure may worsen the condition.

\section{Summary of Recommendations for the Emergency Situation}

Here, we organize the general principles into an approach to difficult mask ventilation based on etiology. Specific situations may be more suited to alternative approaches as previously discussed and summarized in Table 2. These recommendations are intended to simplify and optimize mask ventilation in emergency situations but are not intended to replace the American Society of Anesthesiologists' difficult-airway algorithm. Most importantly, the above recommendations should not delay calling for help, attempting to intubate, or preparing for the emergent invasive airway.

(1) Identify significant leak and compensate: a twohand mask ventilation should be applied without delay using either a bag or ventilator with pressure mode ventilation. An alternative mask size or shape should be employed for the observed mask-patient mismatch. If using a ventilator, looking for collapse of the bellows (or a singlebreath pressure trace if using a ventilator without visible bellows) allows the operator to determine whether the mask seal continues to be inadequate. If an inadequate seal is not fixable, maximize both the oxygen and air flow together over $20 \mathrm{~L} / \mathrm{min}$. If ventilation adequacy improves, oxygenation will improve despite the lower $\mathrm{F}_{\mathrm{IO}_{2}}$. Consider moving to one of several different mask-patient interfaces, as discussed above.
(2) Optimize airway patency: place the patient in the sniffing position, apply jaw thrust and chin lift, and move to a reverse Trendelenburg position. Adjuvant devices (eg, nasal or oral airway) should be employed. Consider paralytics, especially if a return to spontaneous breathing is unlikely. The nasal airway may be more effective than the oral airway particularly for those who have small mouths and large tongues. Ensure that the face mask does not occlude the nose, which frequently occurs during emergency mask ventilation, and maintain CPAP if possible because increased lung volume improves airway patency.

(3) Overcome distal rigidity: the general maneuvers to decrease distal rigidity (if not already undertaken) include reverse Trendelenburg positioning, pharmacologic sedation or paralysis, and increased driving pressures.

\section{Summary}

Despite advances in intubation technology, difficult airways persist. Mask ventilation is a lifesaving skill that should be mastered by clinicians, and a structured algorithmic approach to mask ventilation can be critical to maintaining oxygenation and vitality.

This review provides a logical approach to optimizing mask ventilation based on a comprehensive review of the currently available difficult-airway literature and theory (summarized in Table 2). Inclusion of this type of algorithm in difficult-airway guidelines, training courses, and emergency crisis checklists may result in less morbidity and mortality from failure to ventilate.

\section{REFERENCES}

1. Cheney FW, Posner KL, Lee LA, Caplan RA, Domino KB. Trends in anesthesia-related death and brain damage: a closed claims analysis. Anesthesiology 2006;105(6):1081-1086.

2. Kheterpal S, Han R, Tremper KK, Shanks A, Tait AR, O'Reilly M, Ludwig TA. Incidence and predictors of difficult and impossible mask ventilation. Anesthesiology 2006;105(5):885-891.

3. Han R, Tremper KK, Kheterpal S, O’Reilly M. Grading scale for mask ventilation. Anesthesiology 2004;101(1):267.

4. Orebaugh SL. Difficult airway management in the emergency department. J Emerg Med 2002;22(1):31-48.

5. Wong E, Ng YY. The difficult airway in the emergency department. Int J Emerg Med 2008;1(2):107-111.

6. Miller RD, Eriksson LI, Fleischer, LA, Wiener-Kronish, JP, Young, WL. Miller's anesthesia: expert consult premium edition. Philadelphia: Churchill Livingstone; 2009:361-391.

7. Oto J, Chenelle CT, Marchese AD, Kacmarek RM. A comparison of leak compensation in acute care ventilators during noninvasive and invasive ventilation: a lung model study. Respir Care 2013;58(12): 2027-2037.

8. Oto J, Chenelle CT, Marchese AD, Kacmarek RM. A comparison of leak compensation during pediatric noninvasive ventilation: a lung model study. Respir Care 2014;59(2):241-251.

9. Gerstein NS, Carey MC, Braude DA, Tawil I, Petersen TR, Deriy L, Anderson MS. Efficacy of facemask ventilation techniques in novice providers. J Clin Anesth 2013;25(3):193-197. 


\section{Optimizing Mask Ventilation}

10. Hart D, Reardon R, Ward C, Miner J. Face mask ventilation: a comparison of three techniques. J Emerg Med 2013;44(5):1028-1033.

11. Davidovic L, LaCovey D, Pitetti RD. Comparison of 1- versus 2-person bag-valve-mask techniques for manikin ventilation of infants and children. Ann Emerg Med 2005;46(1):37-42.

12. Joffe AM, Hetzel S, Liew EC. A two-handed jaw-thrust technique is superior to the one-handed "EC-clamp" technique for mask ventilation in the apneic unconscious person. Anesthesiology 2010;113(4): 873-879.

13. Benyamin RM, Wafai Y, Salem MR, Joseph NJ. Two-handed mask ventilation of the difficult airway by a single individual. Anesthesiology 1998;88(4):1134.

14. Bouvet L, Albert ML, Augris C, Boselli E, Ecochard R, Rabilloud $\mathrm{M}$, et al. Real-time detection of gastric insufflation related to facemask pressure-controlled ventilation using ultrasonography of the antrum and epigastric auscultation in nonparalyzed patients: a prospective, randomized, double-blind study. Anesthesiology 2014; 120(2):326-334.

15. Isono S, Eikermann M, Odaka T. Facemask ventilation during induction of anesthesia: how "gentle" is "gentle" enough? Anesthesiology 2014;120(2):263-265.

16. Johnson JO, Bradway JA, Blood T. A hairy situation. Anesthesiology 1999;91(2):595.

17. Vincent C, Ames WA. The bearded airway. Anaesthesia 1998;53(10): 1034-1035.

18. Conlon NP, Sullivan RP, Herbison PG, Zacharias M, Buggy DJ. The effect of leaving dentures in place on bag-mask ventilation at induction of general anesthesia. Anesth Analg 2007;105(2):370-373.

19. Lanier WL. Improving anesthesia mask fit in edentulous patients. Anesth Analg 1987;66(10):1053.

20. Kubota Y, Toyoda Y, Kubota H. Face mask fitting for edentulous patients. Anesth Analg 1993;76(2):450.

21. Rao V, Kosik E, Ganta R, Windrix C. Mask ventilation in edentulous and bearded patients. Anesth Analg 2011;112(6):1513-1514.

22. Elam JO, Titel JH, Feingold F, Weisman H, Bauer RO. Simplified airway management during anesthesia or resuscitation. A binasal pharyngeal system. Anesth Analg 1969;48(2):307-316.

23. Williams WB, Jiang Y. Management of a difficult airway with direct ventilation through nasal airway without facemask. J Oral Maxillofac Surg 2009;67(11):2541-2543.

24. Hillman DR, Platt PR, Eastwood PR. The upper airway during anaesthesia. Br J Anaesth 2003;91(1):31-39.

25. Isono $\mathrm{S}$. One hand, two hands, or no hands for maximizing airway maneuvers? Anesthesiology 2008;109(4):576-577.

26. Isono S, Tanaka A, Tagaito Y, Sho Y, Nishino T. Pharyngeal patency in response to advancement of the mandible in obese anesthetized persons. Anesthesiology 1997;87(5):1055-1062.

27. Tsuiki S, Isono S, Ishikawa T, Yamashiro Y, Tatsumi K, Nishino T. Anatomical balance of the upper airway and obstructive sleep apnea. Anesthesiology 2008;108(6):1009-1015.

28. Tagaito $\mathrm{Y}$, Isono S, Tanaka A, Ishikawa T, Nishino T. Sitting posture decreases collapsibility of the passive pharynx in anesthetized par- alyzed patients with obstructive sleep apnea. Anesthesiology 2010; 113(4):812-818.

29. Tagaito Y, Isono S, Remmers JE, Tanaka A, Nishino T. Lung volume and collapsibility of the passive pharynx in patients with sleepdisordered breathing. J Appl Physiol 2007;103(4):1379-1385.

30. Isono S. Obesity and obstructive sleep apnoea: mechanisms for increased collapsibility of the passive pharyngeal airway. Respirology 2012;17(1):32-42.

31. Liang Y, Kimball WR, Kacmarek RM, Zapol WM, Jiang Y. Nasal ventilation is more effective than combined oral-nasal ventilation during induction of general anesthesia in adult subjects. Anesthesiology 2008;108(6):998-1003.

32. Oto J, Li Q, Kimball WR, Wang J, Sabouri AS, Harrell PG, et al. Continuous positive airway pressure and ventilation are more effective with a nasal mask than a full face mask in unconscious subjects: a randomized controlled trial. Crit Care 2013;17(6):R300.

33. Bennett JA, Abrams JT, Van Riper DF, Horrow JC. Difficult or impossible ventilation after sufentanil-induced anesthesia is caused primarily by vocal cord closure. Anesthesiology 1997;87(5):10701074.

34. Goodwin MW, Pandit JJ, Hames K, Popat M, Yentis SM. The effect of neuromuscular blockade on the efficiency of mask ventilation of the lungs. Anaesthesia 2003;58(1):60-63.

35. Ikeda A, Isono S, Sato Y, Yogo H, Sato J, Ishikawa T, Nishino T. Effects of muscle relaxants on mask ventilation in anesthetized persons with normal upper airway anatomy. Anesthesiology 2012;117(3): 487-493.

36. Warters RD, Szabo TA, Spinale FG, DeSantis SM, Reves JG. The effect of neuromuscular blockade on mask ventilation. Anaesthesia 2011;66(3):163-167.

37. Calder I, Yentis SM. Could 'safe practice' be compromising safe practice? Should anaesthetists have to demonstrate that face mask ventilation is possible before giving a neuromuscular blocker? Anaesthesia 2008;63(2):113-115.

38. Longnecker D. Anesthesiology. New York: McGraw-Hill; 2008:693695.

39. Benumof JL. Laryngeal mask airway and the ASA difficult airway algorithm. Anesthesiology 1996;84(3):686-699.

40. Hauswald M, Ong G, Hun YB, Tan PS. Use of the endotracheal tube as a pharyngeal airway. Am J Emerg Med 1996;14(1):48-49.

41. Kristensen MS. Tube tip in pharynx (TTIP) ventilation: simple establishment of ventilation in case of failed mask ventilation. Acta Anaesth Scand 2005;49(2):252-256.

42. Panadero A, Monedero P, Olavide I, Fernández-Liesa I, Mendieta JM, Macías A. Inflation of the endotracheal tube cuff in the pharynx for ventilation of paralyzed patients with unanticipated difficult airway. Anesthesiology 1999;91(4):1178-1179.

43. Slinger P, Karsli C. Management of the patient with a large anterior mediastinal mass: recurring myths. Curr Opin Anaesthesiol 2007; 20(1):1-3. 